\title{
La autopreparación académica basada en los estilos de aprendizaje de los universitarios
}

\section{Academic selfpreparation based on the learning styles of university students.}

ESPERICUETA-MEDINA， Marta Nieves†̣，SÁNCHEZ-RIVERA， Lilia， VILLARREAL-SOTO, Blanca Margarita y ORTÍZ-TORRES, Vivian

Universidad Autónoma de Coahuila. Facultad de Ciencia, Educación y Humanidades. Saltillo, Coahuila, México.

ID $1^{\mathrm{er}}$ Autor: Marta Nieves, Espericueta-Medina / ORC ID: 0000-0002-4924-4332, Researcher ID Tomson: T-1500-2018, arXiv Author ID: Espericueta2018, CVU CONACYT ID: 372705

ID $1^{\text {er }}$ Coautor: Lilia, Sánchez-Rivera / ORC ID: 0000-0001-9468-2599, Researcher ID Tomson: T-1404-2018, CVU CONACYT ID: 613195

ID $2^{\circ}$ Coautor: Blanca Margarita, Villarreal-Soto / ORC ID: 0000-0001-9314-8001, Researcher ID Tomson: 2357-2018, CVU CONACYT ID: 947979

ID $3^{\text {er }}$ Coautor: Vivian, Ortíz-Torres / ORC ID: 0000-0003-2588-1652, CVU CONACYT ID: 1068920

DOI: $10.35429 / J U P .2020 .11 .4 .16 .23$

Recibido: 20 de Enero, 2020; Aceptado 30 de Junio, 2020

\section{Resumen}

En la presente investigación se abordarán los estilos de aprendizaje en diferentes dimensiones según El Inventory Learning Proceses (ILP), (Schmeck et al., 1977; Schmeck y Ribich, 1978). El objetivo principal es conocer los factores que integran los ejes: rendimiento académico y estilos de aprendizaje de los universitarios de la República Mexicana, además de los atributos que los integran. Se trabajó con una muestra de 253 sujetos. Se procesó la información por medio del análisis descriptivo desde frecuencias y porcentajes, comparativo con prueba $\mathrm{T}$ de Student para grupos independientes y con integracional, análisis factorial exploratorio. Es una investigación cuantitativa, sincrónica y transversal de tipo exploratorio y descriptivo. Entre las principales conclusiones se indica que las características que identifican a los jóvenes relacionados con el género, edad, ciudad, estado, institución, carrera, promedio, entre otras, influyen en el rendimiento académico de los estudiantes. De esta manera, se presentan lo factores que inciden en el aprendizaje de los estudiantes. Se presenta un panorama resumido de la investigación. Una de las conclusiones es que, en tendencia, los estudiantes universitarios poseen un buen rendimiento académico, según el método de estudio, siendo la población de mujeres con más opinión significativa. También se observa que las variables que tienen mayor objetividad las cuales darán una innovación educativa a través de factores. Por último, se presentarán las estrategias innovadoras que han surgido a través del fenómeno de estudio.

Estilos de Aprendizaje, Rendimiento Académico, Métodos de Estudio.

\begin{abstract}
The present investigation, Learning Styles in different dimensions will be addressed according to The Inventory Learning Processes (ILP), (Schmeck et al., 1977; Schmeck and Ribich, 1978). The objective is to know the factors that make up the axes: academic performance and learning styles of the university students of the Mexican Republic, in addition to the attributes that make them up. We worked with a sample of 253 subjects. The information was processed by means of the descriptive analysis from frequencies and percentages, comparative with Student's t-test for independent groups and with integrative, exploratory factor analysis.It is an exploratory and descriptive quantitative, synchronous and transversal investigation $\mathrm{t}$ is indicated that the characteristics that identify young people such as: gender, age, city, state, institution, career, average, among others, influence the academic performance of students. In this way, the factors that affect student learning are presented. A summarized panorama of the research is presented: One of the conclusions is that, in trend, university students have a good academic performance, according to the study method, being the population of women with the most significant opinion. Likewise, it is observed that the variables that have greater objectivity which will give an educational innovation through factors. Finally, the innovative strategies that have emerged through the study phenomenon will be presented.
\end{abstract}

Learning Styles, Academic Performance, Study Method

Citación: ESPERICUETA-MEDINA, Marta Nieves, SÁNCHEZ-RIVERA, Lilia, VILLARREAL-SOTO, Blanca Margarita y ORTÍZ-TORRES, Vivian. La autopreparación académica basada en los estilos de aprendizaje de los universitarios. Revista de Políticas Universitarias. 2020. 4-11:16-23.

\footnotetext{
* Correspondencia al Autor (Correo electrónico: mnieves@uadec.edu.mx)

$\dagger$ Investigador contribuyendo como primer autor.
} 


\section{Introducción}

El aprendizaje se define como una parte importante para el desarrollo de los estudiantes para generar conocimientos significativos a través de diferentes formas dónde se refleja en diferentes factores como el rendimiento académico de los estudiantes, por su relevancia y complejidad. Surgiendo la interrogante de investigación ¿Qué factores integran los ejes: rendimiento académico y estilos de aprendizaje de los universitarios de la República Mexicana? El instrumento que se utilizó para medir el fenómeno de estudio es de un total de 160 reactivos, de los cuales 10 pertenecen a la caracterización de la población y lo que resta será de Estilos de Aprendizaje.

La presente investigación de desarrollo científico es importante porque se observó la información que corresponde a los estilos de aprendizaje basados en la teoría de R. Schmeck (1988), con relación al rendimiento académico de los estudiantes de educación superior de la República Mexicana; de esta manera todos los que forman parte del modelo educativo como son los directivos, administrativos y docentes podrán tomar aquellas decisiones dependiendo a los resultados de esta investigación que pueden ser significativos.

Los principales beneficiaros serán los estudiantes de educación superior de los diferentes Estados de la República Mexicana; ya que con ellos se trabajará esta investigación. Ellos podrán tener acceso a esta información, podrán identificar las ventajas y desventajas que se presentan en su desarrollo de cognitivo referente a su aprendizaje, encontrarán las diferentes estrategias y técnicas que se adapten a la forma de aprender individualmente.

\section{Estilos de aprendizaje}

"Los estilos de aprendizaje son rasgos cognitivos, afectivos y fisiológicos, que sirven como indicadores relativamente estables, de cómo los discentes perciben, interaccionan y responden a sus ambientes de aprendizajes" (Hernández, 2015)
Los estilos se pueden considerar como predisposiciones, relativamente generales $\mathrm{y}$ constantes, que responden a una tendencia del sujeto y derivan de la disposición de un individuo a adoptar la misma estrategia en distintas situaciones, independientemente de las demandas específicas de la tarea. (Lamas, 2015) El enfoque profundo se basa en la motivación intrínseca; el estudiante tiene interés por la materia y desea lograr que el aprendizaje tenga significación personal. Las estrategias se usan para lograr la comprensión y satisfacer la curiosidad personal (Lamas, 2015)

\section{Rendimiento Académico}

La complejidad del rendimiento académico se inicia desde su conceptualización. En ocasiones se le denomina aptitud escolar, desempeño académico o rendimiento escolar, pero, generalmente, las diferencias de concepto solo se explican por cuestiones semánticas ya que se utilizan como sinónimos (Lamas, 2015)

El rendimiento académico se entiende como una medida de las capacidades respondientes o indicativas que manifiestan, en forma estimativa, lo que una persona ha aprendido como consecuencia de un proceso de instrucción o formación. (Lamas, 2015)

El rendimiento varía de acuerdo con las circunstancias, condiciones orgánicas y ambientales que determinan las aptitudes y experiencias (Lamas, 2015)

Incluso, se ha llegado a relacionar con otros conceptos, como desempeño y habilidades académicos. En este sentido resulta pertinente mencionar algunas definiciones de rendimiento académico, realizadas por diferentes autores. (Arlety García Cabezas\& Annia Almeyda Vázquez, 2019)

\section{Metodología por desarrollar}

Partiendo del cuestionamiento ¿Qué factores integran los ejes: rendimiento académico y estilos de aprendizaje de los universitarios de la República Mexicana?, se procede a indagar sobre la bibliografía que da sustento de la propuesta principal de este trabajo, se desarrollan los objetivos, preguntas de investigación e hipótesis de investigación. 
Se modificará un cuestionario de ILP-R versión español el inventario Schmeck (1988), el cual está compuesto de una serie de ítems de afiliación, siendo 150 ítems distribuidos en nueve escalas, algunas de las cuales se dividen a su vez en subescalas para posteriormente ser sometido a la revisión por parte de los sujetos encargados de la supervisión de este trabajo.

La aplicación definitiva se ejecuta vía online, y los datos son obtenidos por medios de recursos electrónicos, de la plataforma digital Forms de Google, con apoyo de la herramienta Excel para su ordenamiento. A dichos datos se transportarán al programa STATISTIC donde se realizarán diferentes tratamientos.

Los datos obtenidos se organizan en una matriz de concentración y se les da tratamiento estadístico para explorar los resultados donde se obtiene un alfa de Cronbach de .92.

Además, se procesó la información en el análisis descriptivo, desde frecuencias y porcentajes, comparativa con prueba $\mathrm{T}$ de Student para grupos independientes y por último integracional, con el análisis factorial exploratorio; es una investigación cuantitativa, sincrónica y transversal de tipo exploratorio y descriptivo.

El instrumento está compuesto por 13 apartados; en el primero se encuentran los datos generales donde se observan variables como: Género, Edad, Ciudad donde realiza sus estudios, Estado dónde realiza sus estudios, Nombre de la institución educativa, Nombre de la carrera Universitaria, Modalidad en que se imparte la carrera, Promedio general, Trabaja actualmente y Ha trabajado con anterioridad.

A partir del segundo apartado hasta el decimotercer apartado corresponden al eje de Estilos de Aprendizaje, medido con 11 variables complejas que constan de 150 simples las cuales son medidas con una escala decimal.

El muestreo es no probabilístico de tipo incidental o de conveniencia; esta es una característica en las investigaciones realizadas por estudiantes y docentes, donde se depende de los permisos de las autoridades escolares para la aplicación del instrumento de medición.

\section{Resultados}

\section{Análisis Descriptivo}

Frecuencias y Porcentajes

A continuación, se presentan las variables que caracterizan al fenómeno estudiado, las cuales son: género, edad, ciudad donde realiza sus estudios, estado donde realiza sus estudios, institución, carrera que cursa, modalidad, promedio, trabaja actualmente y ha trabajado.

Se puede observar la caracterización de la población encuestada, en la variable género, de los datos generales que presenta cuantos estudiantes fueron femeninos y masculinos. Se observó que $67.98 \%$ fueron femeninos y el $32.02 \%$ de masculinos.

La edad que representa a la población es 19años con 22.53\%, después 21años con $19.37 \%$.

La ciudad dónde realiza sus estudios, se observó que el $50.20 \%$ son se Saltillo Coahuila, el $32.61 \%$ son se Acuña Coahuila.

El Estado dónde realiza sus estudios, se observó que el $88.14 \%$ son se Coahuila de Zaragoza, mientras que el $0.40 \%$ pertenecen los Estados del sur del país.

El nombre de la institución educativa dónde realiza sus estudios, de los datos generales que presenta cuantos estudiantes fueron de diferentes instituciones educativas. Se observó que el $30.04 \%$ es el Instituto Tecnológico Superior de Ciudad Acuña, después con un 29.64\% pertenecen a la Universidad Autónoma de Coahuila

La carrera universitaria, muestra que el $22.13 \%$ pertenece a Ingeniería Industrial, el $15.45 \%$ a la Licenciatura en Ciencias de la Educación, el $8.30 \%$ pertenece a la Ingeniería en Gestión Empresarial y el $6.72 \% \%$ a la Licenciatura en Psicología. En la modalidad en que imparte la carrera que cursa, se observa que el $85.38 \%$ de la población tienen la modalidad por semestre, mientras que el $1.19 \%$ es de trimestres. 
El Promedio que se presenta más con un $38.74 \%$ pertenece a el promedio de 90 , después con un $11.46 \%$ pertenece al promedio de $80 \mathrm{y}$ con un mínimo de $0.40 \%$ todos aquellas debajo de 79 de promedio. Los estudiantes que trabajan actualmente son un $34.39 \%$, mientras que los que no trabajan actualmente representan al $65.6 \%$. Los que han trabajado con anterioridad, corresponden a un $77.47 \%$ y los que no han trabajado con anterioridad a un $22.53 \%$.

\section{Análisis Comparativo}

T de Student para muestras independientes

Con la finalidad de comparar muestras a través de sus medias aritméticas y encontrar diferencias significativas, a continuación, se trabajará con un valor probable de error menor a $0.05(\mathrm{p}<0.05)$, los valores estadígrafos que aparecen en este análisis son los valores de la muestra de cada grupo de análisis (Media $(\mathrm{X})$ de los grupos), el valor de la prueba t (t-valor), los grados de libertad (df) y la probabilidad del nivel de error $(p)$.

En el primer comparativo se consideró la variable Género para agrupar a los sujetos de la muestra mientras que las variables dependientes fueron las del apartado de Autoeficiencia organizacional, observando los resultados más significativos encontrados a través del procesamiento de datos, de los cuales destacan la pregunta "Aunque sé que he estudiado cuidadosamente la materia, tengo problemas para recordarla antes del examen" con una diferencia a favor de las mujeres un mientras que los hombres están por debajo. Se infiere que las mujeres a pesar de invertir tiempo al estudio previo a un examen, tienen problemas para recordar la información. Ya que la Autoeficiencia organizacional depende de factores como la serenidad al responder un examen, lo cual impacta de forma negativa en la población femenil mostrándose nerviosas bajo presión.

En la segunda comparación se tomó como variable agrupadora edad y como variable contraste las que conforman el apartado de la variable compleja de Autoeficiencia global, se observa que en la pregunta "Aunque generalmente recuerdo hechos y detalles, encuentro difícil englobarlos dentro de un mismo patrón" se encontró que la media más alta de los que contestaron fueron los de 19 años, es decir los de menor edad.
Se deduce que los estudiantes conforme van creciendo, mejoran sus habilidades cognitivas y memorísticas lo que conforma su Autoeficiencia global. Lo que les representa una dificultad, aun para los jóvenes de 19 años, para los de 22 años ya no lo es, generando patrones de información, además de que presentan una mayor facilidad para localizar los pasajes en el libro de texto ya que es necesario desarrollar habilidades memorísticas y de atención.

En la última comparación se tomó como variable agrupadora el promedio y como variable contraste las que conforman el apartado de la variable compleja de Autoeficiencia global, del resultado del análisis se observa que en la pregunta "La mayoría de mis profesores hablan demasiado rápido" donde la media más alta de los que contestaron fueron los de promedio de 80 , es decir los de promedio más bajo, a diferencia de los de promedio de 99 . Se infiere que los estudiantes conforme desarrollan sus habilidades de comprensión y atención, adquieren la capacidad de seguir el ritmo de sus profesores, lo que impacta de forma positiva y muy notoria en su promedio, construyendo de esta forma su Autoeficiencia global.

\section{Análisis Integracional}

\section{Factorial exploratoria}

Con el propósito de establecer la estructura que subyace en los temas estudiados, se procesa un análisis factorial exploratorio con rotación varimax. La prueba de adecuación muestral de Kaiser-Meyer-Olkin muestra un nivel de adecuación de .01; además la prueba de esfericidad de Barttlet rechaza la hipótesis nula por obtener un nivel crítico (Sig) < 0.05 ; lo cual muestra que la matriz de correlaciones es adecuada para la factorización. Se da lectura a 4 factores de acuerdo con el gráfico de sedimentación donde se presenta el punto de inflexión.

El primer factor denominado Autopreparación explica el $8.12 \%$ de la varianza común del fenómeno estudiado; está integrado por variables complejas de Autoeficiencia Global, Motivación, Autoestima, Autoafirmación, Método de estudio, Procesamiento Profundo Global, Procesamiento Elaborativo, Procesamiento AGENTIC, Manual de Impresiones y Escala de respuestas. 
Se observa que algunos estudiantes que tienen claras sus metas de la educación suelen prepararse con anticipación para los exámenes y no tienen dificultad en localizar determinada información en un texto ya que en su método de estudio realizan esquemas y gráficos para ayudarse, además leen más allá de lo que dicen en clase porque les entusiasma aprender nuevas cosas, normalmente investigan en varias fuentes para entender algún concepto ya que para ellos aprender es divertido por lo cual muestran buena actitud en la escuela, se detienen a reflexionar sobre el tema que leyeron y a menudo critican lo que leen. No encuentran difícil hablar en público, ni tienen miedo de decir a la gente sus sentimientos e ideas o si están en desacuerdo sobre algo, intentan resolver conflictos trabajando por partes un problema desarrollando el pensamiento lógico y sistemático. Se infiere que los estudiantes que muestran interés en su autodesarrollo por medio de métodos de estudio variados y con una actitud positiva en la escuela es porque tienen claras sus metas en la educación lo cual les brinda una autoeficiencia organizacional y una autopreparación académica.

El segundo factor denominado Baja Motivación explica el $9.47 \%$ de la varianza común del fenómeno estudiado; El cual está integrado por variables de Autoeficiencia Global, Motivación, Autoestima, Autoafirmación, Método de estudio, Procesamiento Profundo Global, Procesamiento Elaborativo y Procesamiento AGENTIC.

Se observa que el estudiante que presenta motivación baja tiende a tener problema para organizar mentalmente la información, a pesar de que estudia cuidadosamente tiene problemas para recordar la información en el examen, ya que a menudo se siente confuso ante lo que estudia, lo cual le genera nerviosismo al momento de presentar un examen, tiene dificultad para comprender las explicaciones del profesor por la velocidad con la que capta las ideas, raramente es capaz de inventar procedimientos para resolver un problema ya que tiene problemas para sacar conclusiones lógicas, le resulta difícil encontrar las palabras correctas para expresarse lo que hace que su autoestima sea baja y piense que no es bueno para las tareas escolares.
Respecto a la autoafirmación, el universitario prefiere que el grupo hable antes que hable él, gusta que le digan que va hacer y como lo va hacer, supone que está más interesado en obtener la licenciatura que el contenido. El método de estudio ellos raramente escriben un resumen del material. En su procesamiento Profundo cuando lee una investigación intenta encontrar diferentes modos de interpretar las conclusiones y no le gusta comparar diferentes teorías. En el procesamiento elaborativo sus sentimientos son más importantes sobre su capacidad de decisión o juicio.

Por último, en el Procesamiento AGENTIC el estudiante cuando estudia encuentra dificultad para concentrarse en una sola cosa por lo que normalmente no tienen tiempo para pensar sobre las implicaciones de lo que leen.

Se infiere que los estudiantes que presentan una menor capacidad memorística se les dificulta estudiar, retener información y recordarlo durante un examen, esto afecta en su autoestima de forma negativa, lo cual se refleja en una autoeficiencia global pobre y una baja motivación.

El tercer factor llamado Memorización Positiva presenta un $2.97 \%$ de la varianza común del fenómeno estudiado. Se integra por Autoeficiencia Global, Motivación, Autoafirmación, Métodos de Estudio, Procesamiento Profundo Global, Procesamiento Elaborativo, Procesamiento AGENTIC, Manual de Impresiones y Escala de Respuestas. 
Se observa que los estudiantes al tener buena memoria, presentan una Autoeficiencia global ya que no tienen problemas para recordar información estudiada con anterioridad, son buenos aprendiendo datos como nombre y fechas, lo que les permite responder correctamente en sus exámenes, para ellos su vida está determinada por otras personas, se expresan y se reafirman en la escuela, pueden ayudar a las demás personas a resolver sus problemas sin involucrarse emocionalmente, en el procesamiento agentic el estudiante normalmente memoriza todo lo que tenga que aprender, incluso si no lo entiende, siempre está muy interesado en lo que el profe enseña por lo cual siempre está motivado y con una actitud positiva, nunca olvida los datos aprendidos por lo cual no se aburre, su trabajo es siempre perfecto, nunca ha hecho mal un problema de matemáticas, ni falla en nada que trata de hacer si se lo determina, lo que influye en su Autoafirmación, nunca duda en su actuación gracias a su Autoestima. Se deduce que el estudiante al presentar un Autoeficiencia global positiva muestra un procesamiento agentic de análisis y repetición obteniendo como resultado una memorización positiva de la información.

El cuarto factor denominado Actitud Positiva explica el $2.61 \%$ de la varianza común del fenómeno estudiado; el cual está integrado por variables complejas de Autoeficiencia Global, Motivación, Método de estudio, Procesamiento Profundo Global, Procesamiento Elaborativo $y$ Procesamiento AGENTIC.

Se observa que el estudiante que está motivado para tener éxito en la escuela, no presenta dificultas para planear como abordar una tarea compleja, facilitándole la realización de las tareas escolares así como en prepararse para sus exámenes; tiene claras las metas de la educación, por lo cual estudia para los exámenes manteniendo horarios específicos de estudio, normalmente termina una tarea completamente antes de comenzar otra, siendo ordenado y organizado, al resolver un problema sabe que la clave es el pensamiento lógico y sistemático y ve a los profesores como un guía.
Se deduce que los estudiantes que tienen claras sus metas en la educación adquieren motivación para tener éxito académico lo cual se refleja en la Autoeficiencia organizacional de los Estilos de Aprendizaje que aplican al estudiar lo que les resulta gracias la Actitud positiva que muestran.

\section{Conclusiones}

De acuerdo con los resultados obtenidos de los análisis estadísticos, en este apartado se presentan las conclusiones que muestran la perspectiva de Los Estilos de Aprendizaje de los Universitarios con Actitud Positiva, reflejando un buen Rendimiento Académico, para dar respuesta a la interrogante principal, objetivos e hipótesis se menciona la siguiente:

Se infiere que las mujeres a pesar de invertir tiempo al estudio previo a un examen, tienen problemas para recordar la información. Ya que la Autoeficiencia organizacional depende de factores como la serenidad al responder un examen, lo cual impacta de forma negativa en la población femenil mostrándose nerviosas bajo presión.

Se deduce que los estudiantes conforme van creciendo, mejoran sus habilidades cognitivas y memorísticas lo que conforma su Autoeficiencia global. Lo que les representa una dificultad, aun para los jóvenes de 19 años, para los de 22 años ya no lo es, generando patrones de información, además de que presentan una mayor facilidad para localizar los pasajes en el libro de texto ya que es necesario desarrollar habilidades memorísticas y de atención.

Se infiere que los estudiantes conforme desarrollan sus habilidades de comprensión y atención, adquieren la capacidad de seguir el ritmo de sus profesores, lo que impacta de forma positiva y muy notoria en su promedio, construyendo de esta forma su Autoeficiencia global.

Se infiere que los estudiantes que muestran interés en su autodesarrollo por medio de métodos de estudio variados y con una actitud positiva en la escuela es porque tienen claras sus metas en la educación lo cual les brinda una autoeficiencia organizacional y una autopreparación académica. 
Se infiere que los estudiantes que presentan una menor capacidad memorística se les dificulta estudiar, retener información y recordarlo durante un examen, esto afecta en su autoestima de forma negativa, lo cual se refleja en una autoeficiencia global pobre y una baja motivación.

Se deduce que el estudiante al presentar un Autoeficiencia global positiva muestra un procesamiento AGENTIC de análisis y repetición obteniendo como resultado una memorización positiva de la información.

Se deduce que los estudiantes que tienen claras sus metas en la educación adquieren motivación para tener éxito académico lo cual se refleja en la Autoeficiencia organizacional de los Estilos de Aprendizaje que aplican al estudiar lo que les resulta gracias la Actitud positiva que muestran.

Las variables que integran al fenómeno de estudio en su conjunto son predictoras en su población, por ende, se puede extrapolar en otras poblaciones con las mismas características y semejanzas por lo que se puede tomar decisiones acerca del grupo para ser extrapolada para una población semejante.

Se concluye que los estudiantes universitarios que muestran una autopreparación, es gracias a elaborados sistemas de estudio diseñados por ellos mismos, en los que varían los métodos de estudio gracias a su autoeficiencia, los cuales se basan en sus propias necesidades y sus objetivos académicos, lo que les permite mejorar su confianza en sí mismos y elevar su autoestima. Todo esto se ve reflejado en la actitud positiva que muestran ante los estudios obteniendo así un mejor rendimiento académico y un promedio más alto.
Por lo cual, se propone a las instituciones educativas de nivel superior, implementar desde el tronco común de las distintas carreras, un programa curricular que incluya los diferentes estilos de aprendizaje, desde su teoría hasta la identificación e implementación en cada uno de ellos, según las necesidades individuales de los universitarios, esto como parte de la innovación educativa, cambiando el rol del estudiante de ser solo el receptor a ser más autoeficiente en sus estudios, lo que permitirá potencializar las capacidades intelectuales individuales para así mejorar el rendimiento académico y a su vez preparará a los jóvenes mostrándose siempre competitivos y con una actitud positiva ante los desafíos de la vida a los laboral que les espera.

\section{Referencias}

Arlety García Cabezas\& Annia Almeyda Vázquez. (01 de septiembre de 2019). Rendimiento académico en estudiantes de Psicología. (C. Facultad de Psicología. Universidad de La Habana, Ed.) Scielo. Recuperado el 06 de 06 de 2020, de http://scielo.sld.cu/scielo.php?script=sci_arttext \&pid=S0253-

$92762019000200128 \& \operatorname{lng}=$ es\&nrm=iso\&tlng= es

Cabezas, G. (2018). Rendimiento académico y competencias de investigación en estudiantes de Psicología. (n. d. Psicología, Ed.)

Caso Niebla, J. ; Hernandez Guzman, L. (2014). Investigación Educativa. Red de Revistas Científicas de América Latina y el Caribe, España y Portugal. Obtenido de http://www.redalyc.org/articulo.oa?id=6791020 7

Hernández, H. L. (marzo de 2015). "ESTILOS Y ESTRATEGIAS DE APRENDIZAJE DE LOS ALUMNOS DE LA CARRERA DE PREPARADOR FÍSICO DE LA UNIVERSIDAD TECNOLÓGICA INACAP, SEDE CHILLÁN". Universidad del Bío-Bío Sistema de Bibliotecas - Chile. Recuperado el 06 de 06 de 2020, de http://repobib.ubiobio.cl/jspui/bitstream/123456 789/1188/1/Lopez_Hernandez_Helen.pdf 
Lamas, H. A. (Enero-Junio de 2015). Sobre el rendimiento escolar. (L. P. Academia Peruana de Psicología, Ed.) Revista de Psicología Educativa, 3(1), 313-386. doi:http://dx.doi.org/10.20511/pyr2015.v3n1.74

MINEDU. (2016). Currículo Nacional de la Educación Básica. (Graó, Ed.) Lima perú: Madrid.

Ruiz Ledesma , E. F., Gutiérrez García , J. G., \& Chavarría Báez , L. (2020). Perfiles del estudiante de nivel superior de acuerdo con el desempeño académico y su relación con su entorno socioeconómico . Revista Iberoamericana de Producción Académica y Gestión Educativa, 4.

Schmeck, R. (1982). An introduction to strategies and styles of learning. New York: Plenum Press.

SCHMECK, R., \& RIBICH, F. \&. (1977). Development of a SelfReport Inventory for Assessing Individual Differences in Learning Processes. . Applied Psychological Measurement., 1(3), 413-431. Recuperado el 05 de mayo de 2020

Tapias, M. G. (2018). ESTILOS DE APRENDIZAJE, ESTRATEGIAS PARA ENSEÑAR. SU RELACIÓN CON EL DESARROLLO EMOCIONAL Y "APRENDER A APRENDER". Portal de revistas electrónicas UAM(31). doi:DOI: http://dx.doi.org/10.15366/tp2018.31.004

Valenzuela, A. ; Portillo, S. (2018). La inteligencia emocional en la educación. Educare, 22. 